\title{
La crisis como escenario para surgimiento del emprendimiento social: caso de la Universidad del Valle
}

\author{
The crisis as a scenario for the emergence of social \\ entrepreneurship: the case of the Universidad del Valle
}

Alfonso Rodríguez-Ramírez

Universidad del Valle, Cali, Colombia

alfonso.rodriguez@correounivalle.edu.co

https://orcid.org/0000-0002-8866-1596

\author{
Álvaro Zapata-Domínguez ${ }^{2}$ \\ Universidad del Norte, Barranquilla, Colombia \\ alvaroz@uninorte.edu.co \\ https://orcid.org/0000-0001-8194-2259
}

Recibido: $25-02-20$

Aprobado: 10-06-20

1 Magíster en Ciencias de la Organización.

2 Doctor en Administración. 


\title{
Resumen
}

El propósito del artículo es describir la influencia de los entornos en la evolución histórica de la Universidad del Valle, especialmente en los años 1962-1995 de crisis económica y social, que sirvió de escenario para emprendimientos sociales, liderados por profesores y trabajadores. Estos entornos describieron aspectos específicos sociales, económicos, políticos y culturales, que influenciaron las conductas de líderes comunitarios en el interior de la Universidad para crear empresas sociales con servicios de salud, educación, bienestar social, ahorro y crédito, que la Universidad no podía financiar. Se aplicó una metodología de análisis de contenido, identificando hechos que evidenciaron dificultades de la Universidad para financiar su funcionamiento e inversiones. Se identificó el período 1962-1995 porque, en este período, se establecieron 15 empresas sociales de economía solidaria, que moderaron el impacto de la crisis y dan alternativas de solución a problemas de financiación de la Universidad del Valle.

Palabras clave: crisis económica; entornos; emprendimiento social; líderes comunitarios; Universidad del Valle.

Clasificación JEL: 034

\begin{abstract}
The purpose of this paper is to describe the influence of the environment in the historical evolution of the Universidad del Valle, especially in the years 1962 - 1995 of economic and social crisis, which served as a scenario for social entrepreneurship, led by professors and workers. These environments discovered specific social, economic, political and cultural aspects, which influenced the behavior of community leaders inside the University in order to create social enterprises with services of health, education, social well-being, savings and credit, which the University could not finance. It was applied a methodology of content analysis, identifying facts that showed difficulties for the University to finance its functioning and investment. The period 1962 - 1995 was identified because, in that time, 15 social enterprises of solidarity economy were established, which moderated the impact of the crisis and give alternatives of solution to the problems of the Universidad del Valle financing.
\end{abstract}

Keywords: economic crisis; environments; social entrepreneurship; community leaders; Universidad del Valle. 


\section{Introducción}

El propósito del artículo es describir la influencia de los entornos en la evolución histórica de la Universidad del Valle (Univalle), especialmente en los años 1962-1995 de crisis económica y social, que sirvió de escenario para emprendimientos sociales, liderados por profesores y trabajadores. Por tanto, este artículo trata de elaborar una semblanza histórico-social de emprendimientos sociales que surgieron en Univalle, para comprender su creación y evolución, identificando empresas sociales que lideraron dichos procesos en el período mencionado.

De esta manera, al elaborar la semblanza histórico-social se describen los entornos que muestran aspectos específicos sociales, económicos, políticos y culturales, que influenciaron las conductas de líderes sociales, al interior de Univalle, para crear empresas sociales con servicios de salud, educación, bienestar social, ahorro y crédito, que la Universidad no podía financiar. Por lo tanto, se formula la siguiente hipótesis: los entornos de Univalle conformaron la especificidad de los problemas socioeconómicos que, en conjunto, configuraron su crisis económica, y los entornos sirvieron de escenario para que líderes solucionaran problemas socioeconómicos, mediante la creación de empresas sociales que moderaron su crisis.

El emprendimiento social concebido como "un proceso involucrando el uso innovador y la combinación de recursos para buscar oportunidades para catalizar el cambio social y/o satisfacer necesidades sociales" (Mair y Martí, 2006, p. 37) es relativamente nuevo. Este proceso se enfatiza en el Emprendedor Social como agente de cambio (Dees, 1998; 2007), que se caracteriza por sus rasgos como "Individuos con soluciones innovadoras para los problemas sociales más apremiantes de la sociedad. Ellos son ambiciosos y persistentes, abordando los principales problemas sociales y ofreciendo nuevas ideas para el cambio a gran escala (...)" (Ashoka Foundation, 2015, p. 1).

Perrini y Vurro (2006) dicen que la localización geográfica e histórica de emprendedores es clave para proveer soluciones innovadoras en el manejo de problemas sociales complejos, desde el desarrollo comunitario para la exclusión social y la reducción de la pobreza (Margolis y Walsh, 2003). En este contexto, se identifican los entornos que describen el ambiente social, económico, político y cultural. Estos influencian las acciones de los emprendedores que combinan sus capacidades e iniciativas para describir e identificar las oportunidades en el entorno (Rodríguez, 2009).

El tipo de investigación es cualitativo, con características inductiva y analítica, con fundamento en el análisis histórico. Se usa, además, el estudio cualitativo de casos, definido como una descripción, y un análisis intensivo y holístico de un fenómeno dado por una organización, grupo de personas o un proceso (Merriam, 1988; Yin, 1994); con el propósito de hacer una semblanza histórica, con técnicas comparativas, sobre el modo de ser del Emprendedor Social. Para el artículo se aplicó una metodología de análisis de contenido, identificando hechos que evidenciaron dificultades de la Universidad para financiar su funcionamiento e inversiones.

Entre los resultados, se identifica que el período 1962-1995, en la Univalle, se caracterizó por la influencia del entorno y sus ambientes económico, social, político y cultural, que se combinaron de manera holística y hermenéutica para el surgimiento de, más o menos, unos cincuenta líderes sociales (profesores y trabajadores). Estos fueron influidos por la situación económica; tomaron la iniciativa de aplicar los principios del cooperativismo para crear empresas sociales, que moderaron la crisis económica que vivió la Univalle; identificaron alrededor de 15 empresas sociales privadas con el apoyo de sus directivas, que redujeron el impacto de la crisis y dieron alternativas de solución a problemas financieros de la Univalle.

El artículo se compone de antecedentes de investigación, revisión de literatura, metodología, resultados y discusión, y conclusiones.

\section{Antecedentes}

El estudio sobre crisis y recesiones en el mundo nos dice que, a pesar de que las crisis constituyen la formación de oportunidades para los empresarios, también constituyen el escenario de impactos negativos para el emprendimiento. Así, la Gran Recesión de los años 2007-2009 
tuvo un efecto adverso en el emprendimiento estadounidense (Shane, 2011) y, en general, causó un emprendimiento ralentizado para los países desarrollados y luego para el resto del mundo, paralelamente a la propagación de la crisis (Klapper y Love, 2010). Al final de la recesión, Estados Unidos tenía menos empresas y trabajadores independientes que antes de que comenzara. Algunas fuentes indican que gran parte de la reducción vino del aumento del cierre de empresas, cuyo efecto fue la disminución en la formación de nuevos negocios, particularmente empresas con empleados (Shane, 2011); países desarrollados experimentaron descensos en creación de nuevas empresas (Klapper y Love, 2010).

Adicionalmente, la información mostró que el efecto negativo de la Gran Recesión 20072009 fue mayor para las empresas empleadoras, pues afectó más a los nuevos emprendimientos que a los que no lo eran, y vinculó el autoempleo, afectando, en mayor medida, a las compañías que no lo incorporaron. Para la mayoría de las fuentes disponibles, el efecto de la Gran Recesión en el emprendimiento fue negativo (Shane, 2011).

La literatura hace referencia a la identificación de oportunidades en la crisis, para asumirla con emprendimiento e innovación. Sobre estos aspectos en un contexto de crisis, Peris-Ortiz et al. (2014) dicen que algunos autores consideran que las situaciones de crisis o recesión pueden favorecer el descubrimiento de oportunidades y la innovación. Otros reclaman que la moderación económica tiene un efecto negativo en la actitud emprendedora, reduciendo el descubrimiento de oportunidades y la innovación.

Así, relacionando emprendedores con experiencia, habilidades y conocimientos de gestión de negocios (Kirby, 2003; Krueger, 2007), prácticas de innovación (Hayton, 2005; Lounsbury y Glynn, 2001; Baum y Bird, 2010), actitudes y percepción de oportunidades (Shane y Venkataraman, 2000; Schumpeter, 1934; Cuervo, 2005), en situaciones de crisis económica, se afirma que los empresarios incorporarán el emprendimiento, manteniendo una actitud dinámica. Se mostró relación positiva y significante entre estos empresarios y el desempeño emprendedor en la crisis (Peris-Ortiz et al., 2014).

Además, Devece et al. (2016) indican que las combinaciones de factores emprendedores son fundamentales para impulsar el crecimiento de nuevas empresas en diferentes crisis o recesiones económicas. Utilizando datos de Global Entrepreneurship Monitor (GEM), se identifican dos momentos del reciente ciclo económico de España: la crisis económica de 2008 y el auge económico anterior a esta recesión. El estudio presentó un análisis comparativo cualitativo para identificar las características emprendedoras básicas (innovación y reconocimiento de oportunidades), y los impulsores del emprendimiento (necesidad vs. oportunidad), que aumentan la probabilidad de éxito para nuevas empresas, durante estos dos períodos en el ciclo económico (Devece et al., 2016). Los resultados dicen que el emprendimiento impulsado por necesidades es ineficaz durante las recesiones, y que la innovación y el reconocimiento de oportunidades son más relevantes como factores de éxito durante períodos de crisis que durante la prosperidad (Devece et al., 2016).

Con base en la literatura del emprendimiento en tiempos de crisis económica y social, el contexto de análisis es el emprendimiento social en la Universidad del Valle. Para el caso del emprendedor social, se plantean tres supuestos:

1) Con su experiencia, habilidades y conocimientos de gestión para la toma de decisiones de negocios (proactividad), aumentan el emprendimiento social dentro de un contexto de crisis económica.

2) Con sus prácticas de innovación en productos o servicios, procesos, métodos de comercialización o de organización, prácticas internas, aumentan el emprendimiento social dentro de un contexto de crisis económica, y

3) Con sus actitudes, percepción y reconocimiento de oportunidades (toma de riesgos), aumentan el emprendimiento social en contexto de crisis. 
Por lo tanto, se postula que los emprendedores, en Univalle, asumirán estas prácticas, incorporando el emprendimiento en sentido completo, con ideología cooperativa y manteniendo una actitud emprendedora en situaciones de crisis o recesión económica.

\section{Revisión de literatura}

La crisis como escenario para el análisis del emprendimiento se asocia a la depresión, quiebra o desaparición de empresas, y la pérdida de puestos de trabajo. Se define como la perturbación grave en el sistema económico, sector, industria, clúster u organización, siendo la consecuencia de la acumulación de algún tipo de desequilibrio en el mismo (Rodríguez, 2011). La crisis se caracteriza por la recesión o desequilibrio ocasionado por las pérdidas económicas o falta de liquidez en el mercado, en un entorno nacional o internacional: la más intensa crisis internacional se desarrolló desde 1929 a 1933, y la más cercana es la de 2007-2009, que ocasionaron pérdidas económicas enormes, específicamente en empresas norteamericanas.

La crisis o recesión económica es una situación en la que el crecimiento de la producción o prestación de servicios de una economía es nulo o negativo en varios períodos consecutivos (Fischer, 1992). Las crisis constituyen el escenario de impactos negativos para el emprendimiento o creación de empresas, como motor de desarrollo de la economía (Shane, 2011).

El emprendimiento social concebido como "un proceso involucrando el uso innovador y la combinación de recursos para buscar oportunidades para catalizar el cambio social y/o satisfacer necesidades sociales" (Mair y Martí, 2006, p. 37) es relativamente nuevo. Este proceso enfatiza en el Emprendedor Social como agente de cambio en el sector social (Dees, 1998; 2007), que se caracteriza por sus rasgos como "Individuos con soluciones innovadoras para los problemas sociales más apremiantes de la sociedad. Ellos son ambiciosos y persistentes, abordando los principales problemas sociales y ofreciendo nuevas ideas para el cambio a gran escala (...). Los Emprendedores Sociales líderes son reclutadores en masa de creadores de cambio social" (Ashoka Foundation, 2015, p. 1).

También enfatiza en las ideas del Emprendedor Social como "Individuos con una misión social, capaz de combinar prácticas y conocimiento, así como desarrollar asociaciones para promover el cambio social sostenible" (Macke et al., 2018, p. 677). Se reflexiona sobre el rol del Emprendedor Social en la comunidad, en busca del cambio que permita crear valor social (Dees, 1998).

Sobre la evolución del emprendimiento social, la literatura nos dice que primero surgieron las prácticas sociales para solucionar problemas desde una perspectiva sociológica (Banks, 1972). Sobre los orígenes del emprendimiento social, según El Ebrashi (2013), investigadores debaten sobre cuándo y cómo se implementó el concepto; aunque "se ha mantenido como un concepto controvertido y la evidencia no refleja claramente la forma exacta en que evolucionó" (Hossain et al., 2017, p. 350). Sin embargo, desde el enfoque práctico y concreto, la literatura encuentra que el término "emprendedor social" fue mencionado por primera vez por Banks (1972) en "La sociología de los movimientos sociales". Es decir, el concepto nace de la práctica y los hechos desde la sociología, al analizar las diferentes ideologías, corrientes y movimientos sociales. Hossain et al. (2017) dicen que, aunque no está directamente relacionado con el "emprendimiento", Banks utilizó el término "emprendedor social" para ilustrar habilidades de gestión que procuren resolver problemas sociales (El Ebrashi, 2013).

En este contexto, las prácticas de los emprendedores sociales impactaron las corrientes y movimientos comunitarios, caracterizados por la innovación social y el reconocimiento de oportunidades (Schumpeter, 1934; Dees, 1998; Dees y Anderson, 2006; Drucker, 1985; Shane y Ventkataraman, 2000). Estas prácticas de innovación son realizadas por líderes emprendedores que crean empresa para solucionar problemas sociales y moderar las crisis socio-económicas. 
Perrini y Vurro (2006) dicen que la localización geográfica e histórica de los emprendedores es clave para proveer soluciones innovadoras en el manejo de problemas sociales complejos, desde el desarrollo comunitario para la exclusión social y la reducción de la pobreza (Margolis y Walsh, 2003); como también existen diversos actores innovadores para identificar cómo los protagonistas del Emprendimiento Social ("empresas sociales" y "emprendedores innovadores") sienten y actúan sobre el cambio, en términos de la capacidad de rendimiento alterada de la sociedad (Bornstein, 2004).

En este sentido, Perrini y Vurro (2006) identifican la intervención de líderes en prácticas sociales innovadoras entre los años 1950 y 1980, como Samuel Klein (Casa Bahía, 1952, Brasil); Banco do Nordeste (Crediamigo, 1952, Brasil); Jack Dalton (Pioneer Human Service, 1962, USA); Ted Thibodeau (Thibodeau's Centre for Hearing Health and Communication, 1964, Canadá); Jimmy Pham (Koto, 1966, Vietnam); Ibrahim Abouleish (Sekem, 1968, Egipto); Ed Bullard (Technoserve, 1968, USA); John Maher (The Delancey Street Fundation, 1971, USA); Fazle Hasan Abeb (Bangladesh Rural Advancement Committee, 1972, Bangladesh); Rick Aubry (Rubicon Programs Incorporated, 1973, USA); Govinddapa Venkataswamy (Aravind Eye Hospitals, 1976, India); Muhammad Yunus (Grameen Bank, 1976, Bangladesh); y Bill Drayton (Ashoka Fundation, 1980, USA).

Al analizar cada uno de estos emprendimientos sociales, se observa que se desarrollaron en medio de problemas y crisis económicas coyunturales y/o situacionales, utilizando para ello prácticas sociales innovadoras que solucionaron algunos problemas socioeconómicos y moderaron la crisis (Klein, Abouleish, Yunus, Venkataswamy, Drayton). Los resultados también muestran que la percepción del empresario de las crisis económicas puede ser engañosa en países menos desarrollados, destacando en ellos la experiencia, habilidades y conocimientos, el reconocimiento de oportunidades y la innovación social (Shane y Venkataraman, 2000; Dees y Anderson, 2006; Devece et al., 2016). Al usar la innovación social y reconocimiento de oportunidades, los emprendedores sociales no ven amenazas en crisis económicas, sino escenarios de oportunidades para el emprendimiento (Dees y Anderson, 2006), moderando el impacto de las crisis económicas.

De esta forma, se identifica el papel de la crisis económica y social como un desafío para los emprendedores sociales, con habilidades y conocimientos de gestión y de negocios, con reconocimiento de oportunidades e innovación social. Luego, los emprendedores sociales consideran la relación Crisis-Oportunidades como el escenario mediático y práctico de las soluciones a los problemas sociales, teniendo en cuenta los entornos en los cuales se manifiesta la crisis como punto de partida para el análisis de las oportunidades.

En este sentido, el conocimiento del entorno constituye una fuente de información para la toma de decisiones, identificando oportunidades que se manifiestan en la formación de negocios y/o empresas sociales para la solución de problemas socio-económicos de la sociedad. Así, las teorías económica y organizacional han estado atentas al estudio de los entornos para la formación de la estrategia, en que los emprendedores sociales alinean la solución de problemas sociales y la moderación de la crisis económica en el capitalismo.

\subsection{Importancia del entorno}

La obra de Lawrence y Lorsch (1967; 1987), denominada Empresa y Entorno, constituye un hito en la evolución de las teorías organizacionales y el enfoque de la administración, además de analizar y gestionar las influencias del entorno, que capacita a los empresarios para desafiar un entorno competitivo, en continuo dinamismo y evolución (Rodríguez, 2009).

\footnotetext{
(...) a partir de la teoría de la contingencia y de la concepción de la empresa como sistema integrado, un planteamiento estratégico de la gestión de la organización, que permite las interrelaciones entre entorno, estrategias, estructura, sistemas, tecnología, recursos humanos, capacidades, sistema directivo y pautas de comportamiento, así como las repercusiones que cualquier
} 
modificación en una de ellas origina en las demás, más los consiguientes procesos de cambio y ajuste a que dan lugar (Ilundain, 1987, p. 7).

Sobre los atributos y funciones del entorno, Lawrence y Lorsch (1967) dicen que probablemente no existe un mejor camino para administrar: "Si se comparan organizaciones en diversos entornos, se obtendría una comprensión sistemática sobre los estados de diferenciación e integración que contribuyen al cumplimiento eficaz bajo diferentes condiciones ambientales" (pp. 16-17). Un ejemplo es la gestión del emprendimiento y la responsabilidad social vistas como una capacidad de respuesta ante las necesidades e influencias del entorno.

En el análisis del entorno, se identifican una diversidad de factores externos (económico, social, demográfico, político, tecnológico, jurídico, cultural, geográfico y ecológico) que se manifiestan por medio de la cultura, el lenguaje, la relación con el medio, las relaciones inter-organizacionales y presiones derivadas de la lucha social (Rodríguez, 2009). Los entornos son escenarios que influencian el ámbito organizacional "donde la gestión empresarial debe desarrollarse de manera holística, ecléctica y hermenéutica mediante la continua relación con el entorno, con el propósito de alcanzar resultados que conviertan toda la información del entorno en beneficios económicos y sociales para la organización, con alternativa de crecimiento y sostenibilidad" (Rodríguez, 2009, p. 65).

\subsection{Las empresas sociales según Yunus}

Para analizar las empresas sociales, Yunus (2006) propone inicialmente los emprendedores de negocios sociales, para luego abordar profundamente las empresas sociales. Dice que los emprendedores de negocios sociales pueden desempeñar un papel importante en el mercado, analizando que, en lugar de personas unidimensionales, podemos pensar en un mundo con personas bidireccionales. Para ver cómo funciona la teoría de la firma, se asume que hay dos tipos de emprendedores y cada tipo maximiza una función objetiva separada: un primer tipo, de maximización de beneficios; y un segundo tipo, que no están interesados en la maximización de beneficios, que están impulsados por objetivos sociales, con la creación/apoyo de un tipo especial de empresas.

Yunus (2006) clasifica a los emprendedores sociales en cuatro categorías: sin recuperación de costos, recuperación de algunos costos, recuperación completa de costos y más que la recuperación de costos completos. Una vez que el emprendedor social opera al 100\% o más allá de la recuperación de costos, dice Yunus (2006), se ha "graduado en otro mundo, el mundo de los negocios, con posibilidades de expansión casi ilimitadas.... Este es el momento crítico de una transformación institucional significativa. Se ha mudado del mundo de la filantropía al mundo de los negocios" (pp. 2-3).

En este sentido, Yunus (2006) postula un nuevo mundo de negocios donde las empresas serán de dos tipos:
a) El beneficio bien conocido y bien estable- cido maximizando el tipo, que se dedican a hacer ganancias personales. (Llamémos- los empresas que maximizan el beneficio o PMEs.), y b) Beneficio social maximizando el tipo, que son creados para hacer el bien a las personas, sin prestar atención a la reali- zación de ganancias personales. Los llamo empresas sociales (SBE en inglés) (p. 3).

Para ello, Yunus (2006) da unas características básicas de las empresas sociales:

\footnotetext{
- Una SBE está diseñada y operada como una empresa emprendedora para transmitir todos los beneficios a los clientes. Invierte el principio de maximización de beneficios mediante el principio de maximización de beneficios. En una SBE los beneficios del negocio se transmiten al grupo objetivo, en lugar de traducirlos en beneficios para los inversores.

- Las SBEs son empresas que no son pérdidas y no dividendos. La conclusión para ellos es operar sin incurrir en pérdidas mientras sirven a la gente, y al planeta, particularmente a las personas desfavorecidas, de la mejor manera posible.
} 
- Las SBEs operarán en el mismo mercado con las empresas que maximizan los beneficios, competirán con ellas y tratarán de superarlas por sus cuotas de mercado.

- Las SBEs no solo competirán con las Pequeñas y Medianas Empresas, sino que también competirán con otros SBEs para empujarse mutuamente a mejorar su eficiencia para servir mejor a las personas y al planeta (p. 3).

Para concluir, Yunus (2006) dice que si las empresas sociales (SBE) existen en el mundo real, se les debería dar cabida en nuestro marco conceptual, para capturar la esencia de un ser humano en la teoría. Una vez que las reconozcamos con instituciones, políticas y regulaciones de apoyo, las normas y reglas se establecerán para convertirlas en empresas convencionales.

En el campo de las empresas sociales, en el contexto del capitalismo, Yunus (2008) presenta a la empresa social como un mecanismo complementario de este sistema, que resulta siendo un modelo de empresa innovador y fomenta la ideología de crear empresas para solucionar problemas sociales (específicamente el desempleo y subempleo como efecto de la pobreza), y no para maximizar los beneficios y repartir dividendos. Un ejemplo de empresa social es la alianza Grameen-Danone establecida en Bangladesh para hacer frente a los problemas de desnutrición, desempleo y pobreza, mediante la distribución de Yogurt con alto contenido nutricional para los niños y mujeres (Yunus, 2008).

\section{Metodología}

La investigación utilizó una metodología cualitativa con tendencia inductiva y analítica, con análisis histórico, usando el estudio cualitativo de casos como descripción, y análisis intensivo y holístico de un fenómeno dado por una organización, grupo de personas o un proceso (Merriam, 1988; Yin, 1994); con el propósito de hacer una semblanza histórica con técnicas comparativas sobre el modo de ser del Emprendedor Social. Para el artículo, se aplicó una metodología de análisis de contenido, identificando hechos que evidenciaron dificultades de la universidad para financiar su funcionamiento.

Para el desarrollo del artículo, se aplicaron procedimientos de recopilación tales como la identificación de buscadores (por ejemplo, la ISI Web of Science y Google Academic); la descripción de revistas, autores y artículos; y su clasificación por el concepto de social entrepreneurship and crisis y entrepreneurship and crisis, términos básicos para la recopilación de información. La búsqueda resultó en una cantidad de artículos que, en razón a su amplio número, se leyeron por su abstract. Debido a la novedad relativa del emprendimiento social, se reconoce que sus apariciones en la literatura comienzan a partir de Joseph Banks (1972).

Los criterios de selección de los artículos se relacionan con la escuela de la innovación social, cuyo análisis se dirige al contexto de la innovación y sus repercusiones en el desempeño del emprendimiento social. Además, algunos artículos resaltan sus aspectos y dimensiones: la relación entre emprendimiento, innovación y crisis, y por el análisis de una caracterización del emprendimiento social (Macke et al., 2018).

El proceso de análisis empleado es el análisis de contenido, y consiste en una "técnica de investigación para la descripción objetiva, sistemática y cuantitativa del contenido manifiesto de las comunicaciones con el fin de interpretarlas" (Berelson, 1952, p. 17). En el análisis de contenido de la relación emprendimiento y crisis, se identificó el impacto que puede tener la crisis en el emprendimiento social.

Debido a que es un estudio de caso, el análisis de contenido aplicado en el marco contextual tuvo en cuenta la evolución histórica de la Universidad del Valle y la descripción específica del entorno económico, socio-demográfico, político y cultural, que tuvieron influencia en el ámbito de las decisiones, al interior de la Univalle, para construir una semblanza histórica de la influencia de los entornos en los emprendimientos sociales. 


\section{Resultados y discusión}

El estudio de los entornos para la formación de la estrategia empresarial incluye las organizaciones y, por lo tanto, los emprendedores sociales son el brazo humano para la solución de los problemas de la economía, que "rige la producción y el intercambio de los medios materiales de vida en sociedad" (Engels, 1979, p. 179). Siendo la economía la mano visible en la que se mezclan los entornos social, económico, político, demográfico, cultural, tecnológico, ecológico, jurídico y geográfico (Rodríguez, 2009), que reflejan los problemas económicos y sociales.

En este sentido, se hará énfasis en los entornos que configuraron el contexto de creación y evolución de la Univalle, que nació con problemas estructurales de presupuesto $\mathrm{y}$, posteriormente, sufrió la falta de financiación que dio origen a la crisis económica de los años 1962-1995. De esta manera, se describen los entornos de Univalle como una semblanza histórica para entender los problemas que configuraron la crisis económica, por un lado, y para especificar las soluciones a los conflictos que la moderaron, por otro. Se pretende analizar la influencia de los entornos, en el desarrollo de la crisis económica, como punto de partida para la creación de empresas sociales que moderaron el impacto de dicha crisis.

Para ello se formula una pregunta exploratoria: ¿cómo se caracterizaron los elementos del entorno de la Univalle, durante el período de crisis, que dieron lugar a la formación de Empresas Sociales privadas?

\subsection{La Universidad del Valle}

En el período de análisis 1962-1995, la Univalle se constituyó en el elemento articulador del desarrollo del Departamento del Valle y ha tenido incidencia en toda la región occidental de Colombia. El entorno de Univalle ha sido influenciado por las políticas nacionales de educación superior y el ámbito socioeconómico de la región, que ha incidido en la comunidad universitaria, manifestando la falta de financiación (que, en algunas ocasiones, ha llegado a la crisis económica). Como también los elementos del ambiente internacional: la guerra fría, la revolución cubana y el pensamiento marxista en sus diversas líneas. Estos elementos configuran un pensamiento y entran en juego directamente en la gestación de emprendimientos sociales en Univalle, con la ideología del cooperativismo como alternativa de progreso.

Dentro de este marco, se destacó el papel de Univalle para propiciar condiciones de gestación y desarrollo de empresas sociales, aunque algunas se salgan de los propósitos académicos. Estos hechos expresan una orientación profundamente social frente a necesidades de los miembros de la comunidad universitaria, en el período 1962-1995 (Beltrán, 2002).

Estos hechos y acontecimientos de los entornos económicos, sociodemográficos, políticos y culturales, en Univalle, incidieron de una u otra forma en la creación, formación y evolución de empresas sociales ya mencionadas. Es así como Univalle promueve procesos de cambio social, tecnológico y político, que acentúan la participación de la comunidad universitaria, orientados a buscar el mejor desarrollo para su bienestar (España et al., 1995).

\subsection{Análisis y discusión del entorno de Univalle}

En el análisis del entorno sociodemográfico, económico, político y cultural de Univalle, se observa que son escenarios que configuran una crisis financiera: primero, con el contexto de la creación de Univalle por la falta de mano de obra calificada y el aumento de la migración en los años 1930-1950 (Escobar, 2009); y, segundo, el problema de financiación como universidad pública departamental, que se originó en 19501962, porque no disponía de recursos propios y directos de la nación.

En sus inicios, los emprendimientos sociales creados en Univalle fueron ejemplos de iniciativas exitosas hasta el año 1996, como lo anota Raúl Castro, uno de sus fundadores (Castro, R., comunicación personal, 2019). Parecía que se había logrado combinar el emprendimiento con una orientación social para el beneficio de 
la comunidad universitaria, con el manejo económico adecuado dentro de un entorno determinado por las condiciones de la economía de mercado. Al analizar específicamente los casos de las empresas sociales, varios hechos y acontecimientos obligan a revisar esta impresión y a reflexionar sobre una serie de hechos que fortalecen el emprendimiento social.

\subsection{Emprendimiento social y sector público}

Por ser Univalle una universidad del sector público de orden departamental, donde se crean empresas sociales con el aval institucional de sus directivas para enfrentar la crisis socioeconómica, es un motivo de análisis y reflexión. Se puede decir que la Universidad nació con el problema estructural de generación de ingresos que, con la implementación de la Ley 30 de 1993 y hasta inicios del siglo XXI, se vino solucionando.

En el contexto de los emprendimientos sociales, al analizar los casos de Univalle, según Beltrán (1999), parecería que establecer empresas dentro de instituciones públicas estaría destinado al fracaso. Para ello se plantea la pregunta " ¿Son los casos la prueba de que no se pueden desarrollar empresas exitosas en el ámbito de una universidad pública?" (p. 137). Beltrán (1999) argumenta que:

Primero, porque se han creado varias empresas en el ámbito de la Universidad del Valle que todavía funcionan bien. Entre nuestros casos se encuentran las dos cooperativas de vivienda que han cumplido bien su función, además del Fondo Acumulativo que fue exitoso antes de su transformación en la Fundación de Apoyo.

Segundo, la FES y Solidarios se habían 'liberado’ ya de la Universidad del Valle muchos años antes de entrar en problemas. No se las puede 'acusar' de ser empresas del sector público que han fracasado.

Tercero, hay empresas en Colombia del sector público que son exitosas, como por ejemplo las Empresas Públicas de Medellín. Si se amplía la visión y estudia los casos de empresas públicas en otros países, como por ejemplo en Europa, hay muchos casos de empresas exitosas, con altos niveles de eficiencia y buenas utilidades (p. 137).
La discusión sobre la posibilidad de realizar emprendimiento social, en el ámbito del sector público, tiene mucho para discutir y reflexionar. Varela (1998) muestra cómo ha cambiado en la historia la definición de lo privado, lo público y lo estatal, en que los asuntos son mucho más complejos de lo que parece. ${ }^{3}$ En general, existe una suposición básica de que lo público y lo estatal es lo mismo, se considera el ámbito público como correspondiente a las funciones del Estado. Esta identidad hace que lo privado aparezca como lo particular, que es diferente del Estado. El emprendimiento social nace de la esfera de lo público para solucionar problemas sociales y, en este sentido, hoy en día sus actividades hacen parte del rol de hacedores de política pública.

$\mathrm{Al}$ respecto, Varela (1998) dice que, con el surgimiento del Estado Moderno, se modificaron los criterios: muchas de las dimensiones de la vida social, que tradicionalmente eran propias del ámbito privado, resultaron en el ámbito de lo público. Se asumía la gobernabilidad del Estado como garantía de principios de equidad en contraposición a lo privado, en que la competencia del mercado generaba grandes desigualdades y acumulación de ganancias. Se le asigna al Estado un papel redistributivo y de justicia social. Señala que "todo este proceso de avasallamiento de esfera pública estatal sobre los espacios de la denominada sociedad civil se encuentra en proceso de reversión" (Varela, 1998, p. 10).

Por lo tanto, los emprendimientos sociales surgidos en Univalle son una muestra temprana o adelantada del papel que asumió el Estado o el sector público en la satisfacción de necesidades sociales de la comunidad universitaria, motivando a crear empresas sociales.

\subsection{Influencia del entorno en Univalle}

La caracterización de la influencia del entorno se manifiesta explícita e implícitamente en la cultura organizacional y, específicamente en su ámbito o comportamiento, a través de las

3 La alta complejidad del tema de la dicotomía público-privado se puede ver en los textos de Jeff Weintraub (1997) y Alan Wolfe (1997). 
decisiones tomadas por los diferentes líderes y actores (Zapata y Rodríguez, 2008; 2017). En el caso de la Univalle, esta influencia se expresó en hechos y acontecimientos vividos en su interior, que se describen a continuación.

\subsubsection{Entorno económico}

Desde sus inicios, la Educación Superior ha sido uno de los sectores más abandonados por el Gobierno Central y, por consiguiente, ha tenido un alto déficit presupuestal, más aún con las universidades de orden departamental. Razón por la que Univalle busca el apoyo de entidades privadas para lograr el desarrollo de programas de bienestar social; es así como, en 1953, se creó una Junta Financiera integrada por miembros destacados de las actividades económicas de la ciudad (España et al., 1995). Esta Junta se reunía periódicamente para asesorar al rector en cuestiones financieras.

En este contexto, desde inicios de los años 50 se realizan esfuerzos para que las empresas locales realicen donaciones. Las organizaciones extranjeras mostraron gran interés en apoyar financieramente a la Universidad. "Desde la rectoría del Dr. Carlos Arturo Cabal (1950), se empezaron a conocer las relaciones de la Universidad con la Fundación Rockefeller, pues esta fundación estaba colaborando principalmente en la Facultad de Medicina, en la compra de gran parte de los elementos de laboratorio" (España et al., 1995, p. 47).

Posteriormente, en la rectoría de Mario Carvajal (1954), se empezó una gran campaña para interesar a las industrias y casas comerciales en realizar donaciones a la Universidad, con destinación específica o sin ella, y aliviar así la eterna escasez presupuestal. Aunque estas campañas tuvieron mucho éxito, según los diarios de la ciudad, "fue durante la rectoría del Dr. Alfonso Ocampo Londoño cuando se dio el gran auge especialmente en las donaciones hechas por las fundaciones extranjeras" (España et al., 1995, p. 46).

Según el profesor de la Escuela de Medicina, Guillermo Orozco, debe destacarse la amplia colaboración prestada a la Facultad de Medicina, en 1953-1954, por las Fundaciones Kellog y Rockefeller, mediante la concesión de becas para estudios postgraduados, en Estados Unidos, para varios de los miembros del personal docente. Se observa que "durante el año en curso se había recibido una donación por US\$9.000 con que la Fundación Rockefeller ha deseado colaborar al desarrollo de la Facultad, partida que estuvo designada a la biblioteca; lo que significó gran impulso para el desarrollo de su funcionamiento" (Orozco, 1984, p. 118).

Continuando con la descripción, señala la amplia contribución prestada por las fundaciones, entre 1954-1955, mediante el otorgamiento de becas a personal docente, preparándose para la enseñanza de la Medicina; y, entre 1956-1957, considera el generoso apoyo por parte de las Fundaciones Kellog y Rockefeller.

\footnotetext{
Cuyos representantes han sido valiosos consejeros para la planeación de programas y para la orientación de la Facultad, mediante la concesión de becas a 13 miembros del personal docente y aportes para laboratorios por parte de la primera de ellas, y para 8 docentes por parte de la segunda fundación, aportes para la biblioteca, además de donación de US\$ 504.000 para un programa de 5 años que cubre trabajos de docencia e investigación en el Departamento de Medicina preventiva y salud pública, incluyendo la dotación de la sección de otros y de los laboratorios del Centro de Salud (Orozco, 1984, p. 119).
}

Posteriormente, en materia administrativa, se crean algunos organismos. En 1963, según el Acta No. 25 de junio, el Consejo Directivo aprobó la creación de un organismo de promoción y desarrollo de la Universidad del Valle, el cual tendría funciones específicas destinadas a conseguir la financiación necesaria dentro del país y en el exterior, para el desarrollo de nuevos programas académicos y ensanche de las actuales instalaciones universitarias. Fue así como el 2 de septiembre de 1965 se expidió el Acuerdo No. 7, por el cual se crea la Oficina de Promoción y Desarrollo. Se nombró como director al Dr. Alfonso Ocampo Londoño, quien ocupaba la Decanatura de Estudios (España et al., 1995), con el fin de fortalecer los ingresos. 
De esta manera, la ayuda económica y la asistencia técnica y científica de fundaciones y organizaciones extranjeras -en especial las relaciones de estas con Univalle- constituyen uno de los puntos más delicados y con más trascendencia en su historia, debido a la gran influencia que las organizaciones extranjeras ejercen en la administración de Univalle (España et al., 1995). Ya en 1957, según diarios de la ciudad, Univalle era la única universidad oficial que recibía donaciones de particulares, del comercio y la industria, lo que fue en aumento a partir de 1961. De la importancia y cuantía de esas donaciones, dice el rector Mario Carvajal (1960), en sus alusiones académicas:

\begin{abstract}
La colaboración de las fundaciones extranjeras, la Rockefeller desde hace 13 años, desde hace 5 la Ford, y desde atrás y en importancia media la Kellog, ha sido continua y de gran impulso para la Universidad del Valle. Sin esa cooperación la Universidad se hallaría varios años atrás del hito de desarrollo en que se encuentra (Carvajal, 1960, p. 115).
\end{abstract}

Poco a poco fueron creciendo los ingresos por concepto de donaciones. A raíz de las continuas donaciones, fue necesario crear una fundación que se encargara de administrar los fondos donados a la Univalle. Fue así como surgió la Fundación para el Fomento de la Educación Superior (FES), creada el 30 de noviembre de 1964, como institución de carácter privado sin ánimo de lucro, con fines de adelanto científico y cultural de la educación. Y, en sus comienzos, para brindarle ayuda a Univalle (España et al., 1995).

Así, la FES gestionaría tres propósitos: promoción en la comunidad para conseguir fondos, administración de esos valores y aumentarlos mediante inversiones.

Los aportes de las fundaciones y organizaciones extranjeras, y los de las fuentes nacional o departamental no constituían recursos financieros regulares y generalmente venían con destinación determinada a programas, la FES se ocuparía de lograr el aumento de donaciones provenientes del sector privado. La FES declara que además de promover la imagen de la Universidad, su segunda gran función es la de servir de administradora de los fondos donados a la universidad (Fundación para la Educación Superior, 1964, p. 3).
Posteriormente, en 1967, se planteó la necesidad de crear un fondo vinculado a Univalle para conseguir recursos, como ocurría en la mayoría de universidades en los Estados Unidos, que se manejan como fondos especiales y han sido conformados por donación de egresados y de instituciones externas (España et al., 1995). Para ello, el Dr. Ocampo Londoño, rector de la Universidad, se interesó mucho en la idea, y convencido de ella y de los alcances que obtendría la institución, a largo plazo, en la consecución de recursos financieros, decidió crear el "Fondo de Capitalización de la Universidad del Valle", mediante resolución No. 01 del 14 de febrero de 1967, promulgada por el Consejo Superior. Posteriormente, el 7 de marzo de 1967, se modificó el nombre por "Fondo Acumulativo", el cual tendría carácter permanente y sería manejado por medio de la Fundación para la Educación Superior (FES), de acuerdo con las políticas entre esta institución y Univalle (España et al., 1995).

\subsubsection{Entorno socio-demográfico}

El Valle del Cauca presentaba en 1945 un desarrollo agrícola e industrial rápido, una alta tasa de desempleo, demanda de mano de obra calificada, y un crecimiento demográfico del 5\% (DANE), siendo de los más altos del país. Como respuesta a estos hechos, la Asamblea Departamental fundó la Universidad del Valle que, según el Dr. Ocampo Londoño, "su principal función fue formar a los dirigentes que tendrían la responsabilidad del desarrollo futuro de la región" (España et al., 1995, p. 52). El desarrollo de la misión de la Universidad no solo era transmitir e incrementar el conocimiento, sino actuar además como agente de cambio en la comunidad.

A pesar de que, desde un principio, la principal preocupación de la Universidad del Valle era satisfacer las necesidades de la región, el despliegue de su misión como instrumento de desarrollo regional y nacional solo se definió en la década de los años 70. Es así que, a principios de esa década, el rector Ocampo Londoño señaló que "Las universidades no solo debían establecer severas exigencias académicas, sino que debían obrar en la vida de sus naciones, moviéndose en una acción recíproca de dar y recibir" (España et al., 1995, p. 52). Dentro de la prestación de los servicios sociales de educación, 
se consideraba además que el servicio a la comunidad enriquecía a la docencia e investigación. El Dr. Ocampo Londoño, durante su rectoría, manifestó que los principales objetivos de la universidad eran la "docencia, investigación y servicio a la comunidad, pero tan entrelazados entre sí que para poder cumplir a cabalidad uno de ellos era necesario cumplir los otros dos" (España et al., 1995, p. 53).

De los eventos realizados, durante un simposio organizado en 1966, en la Univalle, en el curso de las diferentes exposiciones se expresó que:

\begin{abstract}
La clase dirigente del Departamento realiza un trabajo de tipo armónico concordantes con las necesidades, lo mismo que con la urgencia de aprovechar al máximo los diversos recursos, donde la Universidad del Valle desde el punto de vista de la educación superior desarrolla planes para mejorar vinculaciones con la comunidad y elaborar proyecciones ambiciosas para incrementar aquel contacto (Ocampo, 1981, p. 36).
\end{abstract}

De esta manera, Univalle, fiel a su derrotero de inversión en la comunidad, presta atención preferencial a la educación, salud, vivienda, crédito y bienestar, busca satisfacer necesidades y problemas sociales en su comunidad universitaria. En este sentido, en 1964, buscando atender las necesidades de vivienda y crédito, un grupo de profesores de la Facultad de Matemáticas se unen y recolectan fondos para un programa de vivienda de la "Alianza para el Progreso", de los Estados Unidos. Entidad que pretendía ayudar a los países subdesarrollados haciendo reservas para solucionar problemas de tipo social (España et al., 1995). El Programa consistía en la construcción del barrio Vipasa, en Cali. Este proyecto no tuvo el apoyo necesario y fracasó, pero como se había conseguido un préstamo de \$20.000 con la Cooperativa Multiactiva Casa Nacional del Profesor (CANAPRO, de Bogotá), se decide hacer préstamos mutuos del fondo a sus dueños. "Es así como empieza a establecerse el Cooperativismo en el Alma Mater, como una posibilidad de que el tipo de organización económica de la Universidad subsista en buenas condiciones, en la lucha inaplazable que se está dando frente al comunismo internacional, siguiendo orientaciones de Estados Unidos" (España et al., 1995, p. 54).

sociedad y economía • e-ISSN: 2389-9050 • No. 41, 2020 • pp. 121-142
La Universidad auspiciaba dos programas para los cuerpos de paz: uno de alto nivel, bajo la responsabilidad de la Facultad de Economía, para asesorar a la pequeña y mediana industria; y otro controlado por el Departamento de Sociología, de jóvenes norteamericanos que vienen como "promotores de desarrollo para ayudar a los campesinos a utilizar los diversos servicios de asistencia técnica agropecuaria, a formar cooperativas, a llevar a cabo construcciones útiles y adoptar innovaciones en el campo agrícola, en salud, nutrición y mejoramiento del hogar" (España et al., 1995, p. 55). Los estudiantes rechazaron el segundo programa. La Facultad de Economía dice que "no es lógico que la Universidad que se queja de falta de profesores, entregue 80 de ellos para 80 muchachos, los cuales ni siquiera iban a la Universidad, que los profesores se desplazaban al lugar donde ellos se encontraban" (Carvajal, 1960, p. 68).

Los estudiantes ocuparon el Departamento de Sociología el 13 de septiembre de 1968, rechazando la intromisión de los norteamericanos en Univalle, que ocupaban profesores que debían estar disponibles para los cursos regulares. Los funcionarios de Univalle calificaron las declaraciones de los estudiantes "por fuera de la realidad de los hechos", puntualizando que "las fundaciones Ford, Kellog, Rockefeller y las demás que contribuyeron al desarrollo del establecimiento, apoyando programas propuestos por la Universidad y la única condición es la de que la planta de profesores contribuya proporcionalmente al mantenimiento de esos programas" (El País, 1968, pp. 1, 19). Estos hechos mostraron que los universitarios adquirieron conciencia antinorteamericana.

Para el año 1971, los estudiantes de la División de Ciencias Sociales y Económicas cuestionaron e impugnaron el plan de estudios porque cada vez los discriminaban y abandonaban más, como "estudiantes rasos" o "marginales", frente a los programas de capacitación de gerentes y administradores. Lo impugnaban porque no era un plan académico que abriera horizontes, sino un programa de empalme con las imposiciones de la empresa privada (España et al., 1995).

El problema consiste en que la Universidad programada por la empresa privada está orien- 
tada a crear con los llamados Fondos Especiales, a través de la Fundación para la Educación Superior FES, un nuevo tipo de investigación $\mathrm{y}$ docencia y un modelo nuevo de profesional. Esta situación condujo a que las relaciones de la FES con la Universidad fueran altamente cuestionadas por los estudiantes y un buen número de profesores, lo que provoca la gran crisis estudiantil en 1971 que interrumpió la mayoría de las actividades de la Universidad. Algunos profesores se retiraron y las relaciones con la comunidad se deterioraron (España et al., 1995, p. 56).

En este contexto, la Universidad "influenciada" por la FES -señalaban los estudiantes- se caracterizaba precisamente porque "suplanta a la comunidad colombiana, por el colonialismo industrial y financiero donde se confunden las necesidades del País y sus perspectivas de desarrollo con la "angustia económica" del grupo de las empresas transnacionales; porque personifica la comunidad en el estamento de la burguesía monopolista asociada al capital foráneo y a su área de dominio" (Ortíz, 1970, p. 74). La Universidad, con estas directrices, presenta una tendencia al elitismo, a la mortalidad académica como fin (Caicedo y Sierra, 1971).

\subsubsection{Entorno político}

Desde los años 60, la Univalle era una institución autónoma y se encontraba aislada de los conflictos políticos, económicos y sociales que vivía el país. La autonomía de la Universidad se ejercía por el Concejo Superior Universitario, constituido por el gobernador o su representante, quien lo presidirá; un delegado del Ministerio de Educación, que deberá ser elegido entre los profesores de la respectiva Universidad; uno de la iglesia, designado por la autoridad pertinente; y por representantes de los profesores, de los estudiantes y corporaciones económicas o asociaciones profesionales o de antiguos alumnos, en el número y forma que determinen los estatutos de la Universidad. Siempre que ese número no sea, en total, mayor de nueve miembros (España et al., 1995).

En este sentido, la participación buscaba la paridad política, que no estaba prescrita en el mandato legal, pero -por un feliz resultado no buscado- el Consejo Superior de la Universidad del Valle enlaza en perfecta paridad, puesto que al representante de la Iglesia (el Obispo) no se podía matricular políticamente; de los ocho restantes, cuatro eran conservadores y cuatro liberales. Además, el aparato político en Univalle estaba formado por elementos extralegales tradicionales como los cabildos abiertos y la organización independiente de los estudiantes (España et al., 1995)

A finales de los años 60 y comienzos de los años 70 , el movimiento estudiantil, organizado junto con un sector de profesores y con el apoyo temporal de algunos grupos gubernamentales, desarrolla una lucha intensa y larga, en gran parte triunfante, contra ese esquema universitario elitista, autoritario y paternalista para volverse más o menos liberal y democrático (Melo, 1978).

Aunque las ayudas de la empresa privada y extranjera tienen su aspecto positivo en programas de desarrollo, representaron un arma de doble filo. Debido a que esos recursos, que tanto necesitaba la Universidad, no podían ser destinados libremente de acuerdo con las necesidades existentes, sino que solo se podían usar en aquellos proyectos que representaban un interés particular para las empresas donantes. Por lo tanto, la autonomía en la toma de decisiones que tenía la Universidad se empezó a ver seriamente afectada (Melo, 1978).

El movimiento estudiantil consideró que las fundaciones extranjeras hacían de Univalle el plan piloto del neocolonialismo, creando la FES, como un sistema de enlace con las fundaciones y corporaciones extranjeras y nacionales asociadas (España et al., 1995). De esta manera, "considera que el gobierno real de la Univalle es la FES, que controla todo el régimen político administrativo y el aparato ideológico-académico, produciendo de hecho una privatización y una desnacionalización de la Universidad que formalmente conserva el gobierno colegiado y el carácter estatal" (Caicedo y Sierra, 1971, p. 79).

Estos movimientos sociales, que estaban marcados por la ideología, preveían una crisis en los años 70, que movilizó a profesores y estudiantes de la Univalle, apuntando hacia una lucha anticapitalista, a la forma de gobierno y la estructura del poder interno y académico, al sistema 
de financiación, a los vínculos y trabas que el capital monopolista provoca; fueron aspectos que respondieron a la crisis (Caicedo y Sierra, 1971). Otro objetivo era la renuncia del Dr. Ocampo Londoño, señalado promotor de una política de autocracia, poder personal, toma de decisiones vertical, y la manera de agenciar, según ellos, los intereses del capitalismo en la vida académica, administrativa y financiera de la Universidad (Caicedo y Sierra, 1971).

Por otro lado, el entorno político reflejó la situación crítica que vivió el Valle del Cauca en lo referente al orden público, en el que estudiantes y profesores protestaron públicamente. Situación que fue relativamente sorteada por algunos gremios y empresarios que estaban convencidos de que los métodos represivos no se podían aplicar para solucionar aquellos hechos que acontecían, en torno a la Universidad, sino que debía ser con métodos democráticos (España et al., 1995).

\begin{abstract}
Los métodos democráticos se impusieron y la Universidad asumió mejor su tarea política dentro del marco general de la sociedad. Entre los efectos que obtuvo el movimiento estudiantil, se pueden destacar la desvinculación de la Fundación para la Educación Superior FES de la Universidad y el retiro del Dr. Ocampo Londoño. Sin embargo los dos aspectos más importantes que motivaron el conflicto no fueron realmente solucionados. Por una parte la cuestión financiera de la Universidad sigue siendo el más grave problema y el gobierno de la Universidad continúa dependiendo del clientelismo, sin que exista una buena relación entre la Universidad y el Estado (España et al., 1995, p. 61).
\end{abstract}

El diario El País (1971, p. 1) señala que, con la renuncia del Dr. Ocampo Londoño en 1971, llega a la rectoría Hugo Restrepo, quien no ejerce su "poder" con plena libertad, ya que es notoria la influencia de un grupo de profesores con ideología comunista. Directivos, decanos y profesores, conservadores y liberales fueron reemplazados por funcionarios de izquierda, con la excusa de un cambio generacional, montando el aparato político-ideológico en Univalle.

\subsubsection{Entorno cultural}

En los años 60, Univalle se caracterizaba por entrar en un período de marcado progreso y crecimiento. Debido a los recursos donados por fundaciones y corporaciones, se introduce el sistema de departamentalización en la institución, se reconstruye y centraliza la biblioteca y se estimula la integración de la investigación, el servicio y la docencia mediante un Comité Central de Investigaciones (España et al., 1995). Se agregan las divisiones de Ciencias, Humanidades, Educación, y Ciencias Sociales y Económicas a la Universidad. La ayuda de la Fundación Ford contribuye al establecimiento de las divisiones de Ingeniería y Ciencias, de la Oficina de Promoción y de la nueva Ciudad Universitaria (Universidad del Valle, 2016).

Con el apoyo de fundaciones extranjeras como Rockefeller, en abril de 1964, con la presencia del escritor y poeta Rafael Maya, se abrió la Biblioteca Mario Carvajal como se conoce hoy. "La Biblioteca fue una de las secciones que recibe especial atención por parte de la Fundación Rockefeller, mas sin embargo, nunca se recibió sugerencia suya en las decisiones sobre la materia, tema, casa editora o idioma para la escogencia de los libros. Se trataba siempre de que la dotación fuera en nuestra lengua materna" (Ortiz, 1970, p. 115).

Continuando con el aspecto cultural, en 1969 se crea la Oficina de Información que, en un principio, se ocupa de asuntos internos, extendiéndose posteriormente la distribución de boletines mensuales a las otras universidades e instituciones, y a personas interesadas en la marcha de la Universidad (España et al., 1995). También suministra información para las campañas de promoción de las actividades universitarias: cursos, investigaciones, conferencias, visitantes, elaborando los folletos respectivos. Para 1969, se encarga de la carta mensual a la oficina de promoción y edita, en colaboración con la organización profesoral, la publicación "Carta del Profesor". Se tuvieron boletines impresos interno y externo el U.V. Noticias Mensual y el U.V. Noticias Petecuy para distribución interna (Beltrán, 1999).

Se observa que, en los aspectos legales, existe una Universidad con un estatuto orgánico único y con autonomía, pero en realidad ocurre que el mecanismo de las donaciones de la empresa privada con sus programas condicionados, constituye una 
forma parcializada para financiar la nómina, al pasar por encima del gobierno formal universitario. Se da prioridad al rendimiento económico, "dejando de lado otros de mayor trascendencia universitaria que incidirían en el cambio social, o sea, que la ayuda extranjera está orientada de tal manera que destruye la función misma de la Universidad, porque produce un desajuste negativo y catastrófico en la relación Universidad - Comunidad" (Caicedo y Sierra, 1971, p. 72).

\subsection{Identificación de necesidades y emprendimientos para la solución}

Hay varios hechos que identifican las necesidades socioeconómicas de profesores y trabajadores, motivando la creación de empresas sociales en la Universidad del Valle. En estas empresas sociales del sector de economía solidaria, se observan algunas características básicas de las SBE de Yunus (2006), como que trasmitieron beneficios a los asociados, no obtuvieron pérdidas y no repartieron dividendos; operan en el mismo mercado que las empresas convencionales, entre otras, alcanzando un buen desempeño.

Un hecho importante que describe la crisis económica por la que atravesaba Univalle, a inicios de los años 60, cuando el rector Mario Carvajal y su equipo analizan la situación de crisis, preguntándose ¿qué hacían para salir de la crisis? Para la época, la Facultad de Medicina tenía convenios con diversas fundaciones norteamericanas como Rockefeller, Kellog y Ford. Aprovechando la visita de Julius Straton, presidente del MIT y miembro de la Junta Directiva de la Fundación Ford, lo invitan a debatir. Una de las alternativas propuestas por el presidente de la Fundación Ford era que las grandes universidades, en los Estados Unidos, tenían una fundación de apoyo por la que recibían donaciones que ayudaban a financiar las actividades académicas, y una oficina de promoción para su divulgación (Lora, 1964). De esta manera, nace la Fundación FES, en 1964, como alternativa para la financiación de los estudios de posgrado de los profesores de la Universidad (como entidad privada sin ánimo de lucro).

De acuerdo a las entrevistas realizadas, los líderes y/o emprendedores sociales manifestaron que la crisis socioeconómica de Univalle se manifestó en diversos tipos de necesidades entre profesores, trabajadores y estudiantes, debido a la carencia de recursos, ingresos y servicios sociales. En los inicios de los años 60, uno de aquellos líderes comentó que algunas veces la universidad se demoraba en pagar dos o tres meses, por la crisis económica por la que atravesaba, teniendo los profesores y funcionarios que recurrir a familiares y amigos para financiar los gastos familiares y de hogar. De esta forma, nace Fetrabuv, en 1964, para los trabajadores y funcionarios, como también nace La Cooperativa Solidarios, en 1964, para los profesores.

Adicionalmente, por las condiciones de crisis económica de Univalle, en 1964, en la Facultad de Medicina, los profesores no eran bien remunerados, ni tampoco tenían la libertad de trabajar en otra Universidad. Situación que llevó a que los pediatras Uriel Estrada y Víctor H. Pinzón se "enamoraran" del cooperativismo, proponiendo la constitución de la Cooperativa de Profesionales Médicos del Valle del Cauca, Coomeva, como alternativa de solución.

Estos son algunos ejemplos de cómo líderes comunitarios, para los años 60, tomaron la iniciativa de crear empresas sociales, fundaciones, cooperativas, fondos de empleados, entre otros, para aminorar el impacto de la crisis económica (por falta de financiación y de presupuesto de Univalle). Algunos líderes comunitarios identifican estas necesidades y plantean soluciones para moderar la crisis, con principios y valores cooperativos como la solidaridad, la empatía, lealtad y participación, proponiendo la creación de empresas sociales (Beltrán, 1999).

Por la crisis socioeconómica que atravesaba, Univalle no fue ajena a los movimientos sociales de los años 60 (Banks, 1972; Acevedo, 2016). Teniendo en cuenta el liderazgo social de algunos profesores y trabajadores, con base en su iniciativa apoyada por las directivas, estudiaron la solución a los problemas que vivían, de necesidades de crédito, vivienda, educación, empleo, salud y bienestar, proponiendo 15 emprendimientos sociales (ver Tabla 1). 
Tabla 1. Listado de emprendimientos sociales y sus emprendedores

\begin{tabular}{|c|c|c|c|}
\hline Necesidades identificadas & Líder o emprendedor social & Emprendimiento social & Año de creación \\
\hline Financiación y salud & $\begin{array}{l}\text { Uriel Estrada Calderón (Q.E.P.D.). } \\
\text { Víctor H. Pinzón Parra y } 26 \text { asociados más. }\end{array}$ & $\begin{array}{l}\text { Cooperativa de Profesionales Médicos del Valle } \\
\text { del Cauca (COOMEVA). }\end{array}$ & 1964 \\
\hline Educación & $\begin{array}{l}\text { Alfonso Ocampo Londoño (Q.E.P.D.). } \\
\text { Gabriel Velásquez Palau (Q.E.P.D.). } \\
\text { Reynaldo Scarpetta. } \\
\text { Pedro Ángel Maya. } \\
\text { Hugo Lora. }\end{array}$ & Fundación para la Educación Superior “FES”. & 1964 \\
\hline Crédito y Financiación & $\begin{array}{l}\text { Ernesto Oliveros. } \\
\text { Víctor H. Pinzón. } \\
\text { James Idrobo. } \\
\text { Stella Gutierrez de Arturo }\end{array}$ & $\begin{array}{l}\text { Casa Nacional del Profesor “CANAPRO”, que } \\
\text { luego sería Cooperativa Financiera Solidarios. }\end{array}$ & 1964 \\
\hline Crédito y bienestar & $\begin{array}{l}\text { José Montoya Gutiérrez. } \\
\text { José María Rivas. } \\
\text { Sergio Elías Ríos. } \\
\text { Francisco Perdomo. }\end{array}$ & $\begin{array}{l}\text { Fondo de Empleados y Trabajadores de la } \\
\text { Universidad del Valle (FETRABUV). }\end{array}$ & 1964 \\
\hline Financiación & $\begin{array}{l}\text { Alfonso Ocampo Londoño (Q.E.P.D.). } \\
\text { Gabriel Velásquez Palau (Q.E.P.D.). } \\
\text { Hugo Lora. } \\
\text { José Serna. }\end{array}$ & $\begin{array}{l}\text { Fondo de Capitalización de la Universidad del } \\
\text { Valle (ya desaparecido). Después fue Fondo } \\
\text { Acumulativo de Univalle. }\end{array}$ & 1967 \\
\hline Salud & $\begin{array}{c}\text { Edgar Torres. } \\
\text { Armando González (prohombre). } \\
\text { Andrés Echeverry. } \\
\text { Antonio Reyes. } \\
\text { Arnoldo Levy. }\end{array}$ & Centro Médico IMBANACO. & 1970 \\
\hline Salud & $\begin{array}{l}\text { Martin Wartenberg. } \\
\text { Álvaro Garcés. }\end{array}$ & Fundación Valle del Lili. & 1971 \\
\hline Vivienda & $\begin{array}{l}\text { Jorge Lozada. } \\
\text { Edgar Bejarano. }\end{array}$ & Cooperativa Puente Palma I. & 1971 \\
\hline Financiación & $\begin{array}{l}\text { Grupo de docentes. } \\
\text { Fernando Naranjo. } \\
\text { Víctor H. Pinzón. }\end{array}$ & $\begin{array}{c}\text { Fondo de Empleados Docentes de la } \\
\text { Universidad del Valle (FONVALLE). Ligado a } \\
\text { CANAPRO. }\end{array}$ & 1978 \\
\hline Vivienda & $\begin{array}{l}\text { Roberto Behar. } \\
\text { Mario Yepes. } \\
\text { Álvaro Zapata. }\end{array}$ & Cooperativa Puente Palma II. & 1980 \\
\hline Bienestar & $\begin{array}{l}\text { Camilo Bautista (QEPD). } \\
\text { Pedro Supelano (QEPD). } \\
\text { Jorge Calle (QEPD). } \\
\text { Alirio Sardi (QEPD). } \\
\text { Raúl Castro. }\end{array}$ & $\begin{array}{c}\text { Corporación de Profesores de la Universidad } \\
\text { del Valle, Club Piedralinda. }\end{array}$ & 1985 \\
\hline Crédito y bienestar & $\begin{array}{l}\text { Harold José Rizo. } \\
\text { Teresa de Padilla. } \\
\text { William Escobar. } \\
\text { Roberto Ospina. } \\
\text { Rubén Darío Echeverry. }\end{array}$ & $\begin{array}{l}\text { Cooperativa de Egresados de la Universidad del } \\
\text { Valle -COOUNIVALLE-(Antes Asociación de } \\
\text { egresados de Economía). }\end{array}$ & 1985 \\
\hline Empleo & $\begin{array}{l}\text { Jaime Enrique Galarza. } \\
\text { Rubén Darío Echeverry. } \\
\text { Oscar Rojas. }\end{array}$ & Fundación de Apoyo a la Universidad del Valle. & 1992 \\
\hline Inversión & $\begin{array}{l}\text { Max Enrique Nieto (QEPD). } \\
\text { Alirio Sardi (QEPD). } \\
\text { Rubén Darío Echeverry. }\end{array}$ & PROMIVALLE. & 1990 \\
\hline Vivienda & $\begin{array}{l}\text { Alirio Sardi (QEPD). } \\
\text { Álvaro Orozco. } \\
\text { Édgar Díaz. } \\
\text { Gabriel Cárdenas. }\end{array}$ & $\begin{array}{l}\text { COOVICEDROS. } \\
\text { Piedra Grande. }\end{array}$ & 1994 \\
\hline
\end{tabular}

Fuente: elaboración propia. 


\section{Conclusiones}

Respondiendo las preguntas iniciales sobre los entornos (económico, socio-demográfico, político y cultural), y su influencia en la creación de emprendimientos sociales, como una moderación del impacto de la crisis socioeconómica, se encontró que se trata de iniciativas con propósitos sociales. En estas, Univalle aparece como inspiradora y motivadora inicial, que mueve a la acción a sus líderes comunitarios, apoyados por las directivas de la Universidad. En este sentido, se puede decir que los líderes identificaron oportunidades del entorno para ralentizar o moderar el impacto de la crisis, por medio de proyectos y empresas sociales, establecidas dentro de la Universidad y asumidas como institucionales por el apoyo de las directivas (España et al., 1995). La Univalle fue, de hecho, la única universidad donde se establecieron emprendimientos de carácter privado.

$\mathrm{Al}$ reflexionar sobre los resultados y el impacto que tuvieron los emprendimientos sociales, en Univalle, se analizaron algunas señales como el rol de las empresas sociales que moderaron el impacto de la crisis socioeconómica. Se justifica que "la mayoría de los líderes sociales querían participar activamente en proyectos de bienestar social. Y que además de servir a la comunidad universitaria les daba un sentido de pertenencia e identidad" (Beltrán, 1999, p. 134).

Luego, la motivación de los líderes o emprendedores sociales era el bienestar colectivo de la comunidad universitaria. Haciendo un rastreo por las motivaciones, los pensamientos expresados por los líderes, la organización de la gestión, los estatutos, y las actividades realizadas por las empresas en sus inicios.

Consideramos que sí existe un tipo social de espíritu empresarial dónde los intereses están centrados en el bienestar de la comunidad. El énfasis en el interés colectivo confirma algunas de las características del espíritu empresarial presentadas en el marco teórico y que hacen parte de los perfiles de empresarios analizados en la teoría. El rastreo también muestra que muchas de las características consideradas como 'individualistas', que tradicionalmente apare- cen como esenciales en las discusiones, no son importantes o no son mencionadas en nuestros seis casos (Beltrán, 1999, p. 134).

Los líderes o emprendedores sociales tenían intereses originales que proyectaron hacia la comunidad universitaria. En este sentido, "Intereses tales como perfilarse como líder en su comunidad para llevar a cabo proyectos colectivos, combinan los intereses de logro individual con el enfoque hacia acciones que benefician a la comunidad universitaria o a la región" (Beltrán, 1999, p. 134).

Otra característica de los emprendedores sociales, en Univalle, se describe en aspectos que tienen que ver con la gestión de la comunidad, como el trabajo en grupo, las acciones de coordinación y las acciones intergrupales, y que fueron altamente valorados en sus actividades.

\section{Hay interés en participar activamente en la vida universitaria con proyectos colectivos, de satisfacción de necesidades básicas como en los casos de Cooperativa de Vivienda para la comunidad universitaria y de bús- queda de soluciones para el problema fi- nanciero de la Universidad del Valle en los otros casos (Beltrán, 1999, p. 135).}

El análisis del entorno sirvió para la identificación de oportunidades por parte de líderes, como emprendedores sociales, con el apoyo de las directivas de Univalle.

\begin{abstract}
Podemos decir que existió un entorno favorable en la Universidad del Valle dónde la institución apoyó decididamente estas prácticas emprendedoras, a través de la prestación de personal, oficina y ayudas administrativas, de permitir las actividades dentro de las horas de trabajo de los líderes, de respaldo frente a instituciones estatales, etc. (Beltrán, 1999, p. 135).
\end{abstract}

El entorno político de la época (1960-1990) estaba muy polarizado por las ideologías capitalistas y comunistas, de derecha y de izquierda, que dieron lugar a enfrentamientos, conflictos y problemas de poder que asumieron los partidarios de ambas ideologías. Se reconoce un pensamiento izquierdista en muchos de sus líderes, influidos por los movimientos políticos, 
las revoluciones socialistas, las prácticas cooperativistas, es decir:

\begin{abstract}
Estos valores iban ganando fuerza, se confiaba en las posibilidades de la unión, en la fuerza de la colectividad para lograr proyectos exitosos, en trabajar para el bien común. Se soñó con sociedades más igualitarias, en fin, el auge del pensamiento socialista era parte de los valores vigentes de la época a finales de los años 1960s e iniciando los 1970s del siglo XX. Más recientemente se puede notar un cambio de valores hacia tendencias más individualistas, como por ejemplo la competencia, la ganancia personal, el ascenso laboral y social, valores que forman parte de un pensamiento llamado 'neoliberal' (Beltrán, 1999, p. 135).
\end{abstract}

En total, se crearon cerca de 15 empresas sociales que moderaron el impacto de la crisis socioeconómica, con el propósito de satisfacer necesidades de vivienda, educación, empleo, y bienestar social, enriqueciendo la comprensión sobre organizaciones diversas de carácter social y de su entorno para la identificación de oportunidades en Univalle.

El conocimiento del proceso de formación, las experiencias en sus comportamientos, los cambios de los valores de sus líderes que se manifiestan en comportamientos empresariales determinados, los actores externos que influyen en estas organizaciones, nos comprueban la alta complejidad del desarrollo organizacional. Muestra además la fuerte influencia del entorno, a través de la cultura organizacional -en nuestro caso, la de la Universidad del Valle- de políticas macroeconómicas, de condiciones políticas mundiales como por ejemplo la 'guerra fría, de políticas de educación superior, etc., que hasta pueden llegar a ser decisivas para el destino de una organización empresarial (Beltrán, 1999, p. 136).

Este análisis concluye que la crisis vivida por Univalle mostró el escenario para el fomento de iniciativas empresariales con carácter social y cooperativista, en que puede existir y tener éxito económico un tipo de emprendimiento social que se diferencia profundamente de la visión del empresario individualista, egoísta y exclusivamente orientado hacia las utilidades económicas, que domina el pensamiento empresarial en la economía de mercado (Yunus, 2006).

En las empresas sociales surgidas en la Universidad del Valle, entre 1962-1995, se observaron algunas características básicas de las SBE de Yunus (2006), como la transmisión de beneficios a los asociados (Coomeva, Solidarios, Fonvalle, Fetrabuv, Puente Palma I y Puente Palma II), no obtuvieron pérdidas y no repartieron dividendos (Centro Médico Imbanaco, Fundación Valle del Lili, Coomeva, Corpruvalle-Piedralinda), y operan en el mismo mercado que las empresas convencionales (Coomeva, Solidarios, Centro Médico Imbanaco, Fundación Valle del Lili). Algunas han alcanzado buen desempeño como empresas sociales (SBE), a tal punto que hoy se encuentran creciendo y consolidándose (Yunus, 2006), no solo como las mejores de Colombia, sino en Latinoamérica (Coomeva, Fundación Valle del Lili, Centro Médico Imbanaco).

\section{Referencias}

Acevedo, A. (2016). Ideología revolucionaria y sociabilidad política en los grupos universitarios maoístas de los años 60 y 70 en Colombia. Historia Caribe, XI(28), 149-175. http://dx.doi.org/10.15648/ hc.28.2016.6

AshokaFoundation.(2015).WhatareSocialentrepreneurs?http://www.ashoka.org/social_entrepreneur

Banks, J. A. (1972). The Sociology of Social Movements. Springer Edition. https://link.springer.com/ book/10.1007/978-1-349-01452-1

Baum, J. R. y Bird, B. J. (2010). The successful intelligence of high-growth entrepreneurs: links to new venture growth. Organization Science, 21(2), 397-412. https://doi.org/10.1287/orsc.1090.0445 
Beltrán, D. (1999). El espíritu empresarial en la Universidad del Valle (tesis de maestría). Universidad del Valle, Cali, Colombia.

Beltrán, D. (2002). El espíritu empresarial en la creación de organizaciones de carácter social en la Universidad del Valle (documento de trabajo inédito). Universidad del Valle.

Berelson, B. (1952). Content Analysis in Communication Researches. Free Press. https://doi. org/10.1177/000271625228300135

Bornstein, D. (2004). How to change the world. Social entrepreneurs and the power of new ideas. Oxford University Press.

Caicedo, S. y Sierra, A. (1971). Crisis Universitaria en Colombia. El tigre de papel.

Carvajal, M. (1960). Testimonio universitario. Biblioteca Universidad del Valle.

Cuervo, A. (2005). Individual and environmental determinants of Entrepreneurship. International Entrepreneurship and Management Journal, (1), 293-31l. https://doi.org/10.1007/s11365-005-2591-7

Dees, J. G. (1998). The meaning of social entrepreneurship. www.gsb.stanford.edu/services/news/ DeesSocentrepPaper.html

Dees, J. G. (2007). Taking social entrepreneurship seriously. Society: Transaction Social Science and Modern, 44(3), 24-31. https://doi.org/10.1007/bfo28199236

Dees, J. G. y Anderson, B. B. (2006). Framing a Theory of Social Entrepreneurship: Building on Two Schools of Practice and Thought. Research on Social Entrepreneurship, ARNOVA Occasional Paper Series, 1(3), 39-66.

Devece, C., Peris-Ortiz, M. y Rueda-Armengot, C. (2016). Entrepreneurship during economic crisis: Success factors and paths to failure. Journal of Business Research, 69(11), 5366-5370. https://doi. org/10.1016/j.jbusres.2016.04.139

Drucker, P. F. (1985). Innovation and Entrepreneurship. Harper \& Row.

El Ebrashi, R. (2013). Social entrepreneurship theory and sustainable social impact. Social Responsibility Journal, 9(2), 188-209. https://doi.org/10.1108/SRJ-07-2011-0013

El País. (1968, 14 de septiembre). Ningún acuerdo en la "U”. El País, pp. 1 y 19.

El País. (1971, 21 de septiembre). ¿Quién manda en la U? El País, p. 1.

Engels, F. (1979). Anti-Dühring. Editorial Pueblo y Educación.

Escobar, G. (2009). La población en Santiago de Cali: Siglo XX y primera década del siglo XXI. Departamento Administrativo de Planeación de Santiago de Cali.

España, S. E., Guerrero, S. I., Olaya, F. M., Ortega, L. M., Rodríguez, O. L. y Santos, J. C. (1995). 50 años de Espíritu Empresarial Social en la Universidad del Valle: Una mirada desde la perspectiva de la cultura organizacional. Tomo I y II. Facultad de las Ciencias de la Administración.

Fischer, K. (1992). Diccionario empresarial. Limusa.

Fundación para la Educación Superior. (1964). Acta Creación de FES. Archivo FES Cod. 142 CI-0013. 
Hayton, J. C. (2005). Promoting corporate entrepreneurship through human resource management practices: A review of empirical research. Human Resource Management Research, 15(1), 21-41. https://doi.org/10.1016/j.hrmr.2005.01.003

Hossain, S., Saleh, M. A. y Drennan, J. (2017). A critical appraisal of the social entrepreneurship paradigm in an international setting: a proposed conceptual framework. International Entrepreneurship Management Journal, (13), 347-368. https://doi.org/10.1007/s11365-016-0400-0

Ilundain, J. M. (1987). Prólogo. En P. Lawrence y J. Lorsch (Eds.), La Empresa y su Entorno (pp. 1-13). Plaza y Janes Editores, S.A.

Kirby, D. A. (2003). Entrepreneurship education: can business schools meet the challenge. En E. Genesca, D. Urbano, J. L. Capelleras, C. Guallarte y J. Vergès (Eds.), Creación de empresas y emprendimiento (pp. 359-375). Servei de publicacions de la Universitat Autonoma de Barcelona.

Klapper, L. y Love, I. (2010). The impact of the financial crisis on New Firm Registration. Policy Research Working Paper 5444. The World Bank, Development Research Group.

Krueger, N. F. (2007). What lies beneath? The experiential essence of entrepreneurial thinking. Entrepreneurship Theory and Practice, 31(1), 123-138. https://doi.org/10.1111/j.1540-6520.2007.00166.x

Lawrence, P. y Lorsch, J. (1967). Organization and Environment. Harper Raw.

Lawrence, P. y Lorsch, J. (1987). La Empresa y su Entorno. Plaza \& Janes Editores, S.A.

Lora, H. (1964). Informe Fundación FORD (pp. 5-6). Archivo FES

Lounsbury, M. y Glynn, M. A. (2001). Cultural entrepreneurship: stories legitimacy and the acquisition of resources. Strategic Management Journal, (22), 545-564. https://doi.org/10.1002/smj.188

Macke, J., Sarate, J. A. R., Domeneghini, J. y Da Silva, K. A. (2018). Where do we go from now? Research framework for social entrepreneurship. Journal of Cleaner Production, 183, 677-685. https://doi. org/10.1016/j.jclepro.2018.02.017

Mair, J. y Martí, I. (2006). Social entrepreneurship research: A source of explanation, prediction, and delight. Journal of World Business, 41, 36-44. https://doi.org/10.1016/j.jwb.2005.09.002

Margolis, J. D. y Walsh, J. P. (2003). Misery loves companies: Rethinking social initiative by business. Administrative Science Quarterly, 48(2), 268-305. https://doi.org/10.2307/3556659

Melo, J. O. (1978). Consideraciones sobre la situación universitaria. Revista Colombiana de Educación, (2), 1-7. https://doi.org/10.17227/01203916.4965

Merriam, S. B. (1988). Qualitative research in practice: examples for discussion and analysis. Jossey-Bass.

Ocampo, J. A. (1981). El Desarrollo Económico de Cali en el Siglo XX. En J. A. Ocampo, Santiago de Cali - 450 años de historia (pp. 10-41). Alcaldía de Cali.

Orozco, G. (1984). Escuela de Medicina y su Universidad. Universidad del Valle.

Ortíz, E. (1970). Universidad del Valle 1945-1970. Universidad del Valle.

Peris-Ortiz, M., Fuster-Estruch, V. y Devece-Carañana, C. (2014). Entrepreneurship and innovation in a context of crisis. En K. Rüdiger, M. Peris-Ortiz y A. Blanco-González (Eds.), Entrepreneurship, innovations and economic crisis. Lesson for research, policy and practice (pp. 1-10). Springer. 
Perrini, F. y Vurro, C. (2006). Social entrepreneurship: Innovation and social change. Across theory and practice. En J. Mair, J. Robinson y K. Hockerts (Eds.), Social entrepreneurship (pp. 57-85). Palgrave-MacMillan. https://link.springer.com/chapter/10.1057/9780230625655_5

Rodríguez, A. (2009). Influencia del entorno en el desarrollo del direccionamiento estratégico. Cuadernos de Administración, 25(42), 63-80. https://doi.org/10.25100/cdea.v25i42.414

Rodríguez, C. E. (2011). Diccionario de Economía. Etimológico, conceptual y procedimental. Universidad Católica.

Schumpeter, J. A. (1934). The theory of economic development: An inquiry into profits, capital, credit, interest, and the business cycle (Vol. 55). Transaction Publishers.

Shane, S. (2011). The Great Recesion's effect on entrepreneurship. Economic Commentary, (04), 1-4.

Shane, S. y Venkataraman, S. (2000). The promise of entrepreneurship as a field of research. Academic Management Review, 25(1), 217-226. https://doi.org/10.5465/amr.2000.2791611

Universidad del Valle. (2016). Reseña Histórica. http://www.univalle.edu.co/

Varela, E. (1998). Desafíos del interés público - identidades y diferencias entre lo público y lo privado. Programa Editorial Universidad del Valle.

Weintraub, J. (1997). The Theory and Politics of the Public-Private Distinction. En J. Weintraub y K. Kumar (Eds.), Public and Private in Thought and Practice (pp. 1-42). The University of Chicago Press.

Wolfe, A. (1997). Public and Private in Theory and Practice: Some Implications of an Uncertain Boundary. En J. Weintraub y K. Kumar (Eds.), Public and Private in Thought and Practice (pp. 182203). The University of Chicago Press.

Yin, R. K. (1994). Case Study Research. Design and Methods. Sage.

Yunus, M. (2006). Social Business Entrepreneurs are the Solution. Skoll World Forum on Social Entrepreneurship, Oxford University, Oxford, Reino Unido.

Yunus, M. (2008). Un Mundo Sin Pobreza: Negocios Sociales y el Futuro del Capitalismo. Public Affairs.

Zapata, A. y Rodríguez, A. (2008). Gestión de la Cultura Organizacional. Bases conceptuales para su implementación. Universidad del Valle.

Zapata, A. y Rodríguez, A. (2017). Gestión de la Cultura Organizacional. Programa Editorial de la Universidad del Valle. 